В.И. Носков, Н.В. Мезенцев, Г.В. Гейко, В.И. Панченко

Национальный технический университет «ХПИ», Харьков

\title{
ОБРАБОТКА СИГНАЛОВ В БОРТОВОЙ СИСТЕМЕ УПРАВЛЕНИЯ ДИЗЕЛЬ-ПОЕЗДА ДЭЛ-02
}

Показано, что наличие упругих связей в системе передачи момента, а такюе постоянно изменяющихся условий сиепления колеса с рельсом приводят к колебаниям на выходе датчика частоты вращения ротора. Проведён анализ различных способов сглаживания этих сигналов. Приемлемым оказалось сглаживание сигналов путём вычисления простого скользящего среднего. Предлагается обработка сигналов, получаемых с датчиков частоты вращения двигателей в тяговом асинхронном приводе.

Ключевые слова: датчик частоты вращения, тяговый асинхронный привод, система передачи момента, обработка сигналов.

\section{Введение}

Постановка проблемы и анализ литературы. Одним из важнейших направлений технического развития железнодорожного транспорта является внедрение в тяговых приводах (ТП) трёхфазных асинхронных двигателей. ТП с тяговыми асинхронными двигателями (ТАД) обладает лучшими технико-экономическими показателями по сравнению с ТП с двигателями постоянного тока $[1,2]$. В отечественном дизель-поезде ДЭЛ-02 применяется ТП с ТАД.

Структурная схема ТП моторного вагона дизель-поезда приведена на рис. 1.



Рис. 1. Структурная схема ТП моторного вагона дизель-поезда ДЭЛ-02: Д - дизель, СГ - синхронный генератор, ВУ - выпрямительная установка, АИН1, АИН2 - автономные инверторы напряжения, ТАД1, ТАД2 - тяговые асинхронные двигатели, ДЧВ1, ДЧВ2 - датчики частоты вращения

Создание современного и экономически эффективного электропривода для тягового подвижного состава потребовало разработки микропроцессорной системы управления, что, в свою очередь, привело к необходимости применения новых средств измерений и обработки сигналов. К числу таких сигналов относится частота вращения ротора ТАД.

Информация по частоте вращения ротора даёт возможность определять неисправности ТАД, реализовать систему автоматического регулирования (CAР) тягового электропривода, обеспечить защиту от боксования и юза и др. На дизель-поезде ДЭЛ-02 с ТАП измерение частоты вращения ротора ТАД выполнено с помощью ДЧВ, состоящего из шестерни с большим числом зубьев, которая установлена на валу ТАД, и чувствительного элемента - датчика, использующего эффект Холла. Вращающий момент от ТАД к колёсной паре передаётся через ряд упругих элементов (рис. 2).

Наличие упругих связей в системе передачи момента, а также постоянно изменяющихся условий сцепления колеса с рельсом, могут вызывать колебания тягового момента, которые передаются на ротор ТАД и приводят к колебаниям сигнала частоты на выходе ДЧВ. Поскольку к сигналу предъявляются достаточно высокие требования по качеству, быстродействию и разрешающей способности, возникает необходимость применения обработки данного сигнала.

Целью статьи является исследование на математической модели системы привода отечественного дизель-поезда ДЭЛ-02 для оценки колебаний частоты вращения ротора ТАД, а также разработка эффективного средства для сглаживания выходной частоты ДЧВ. 


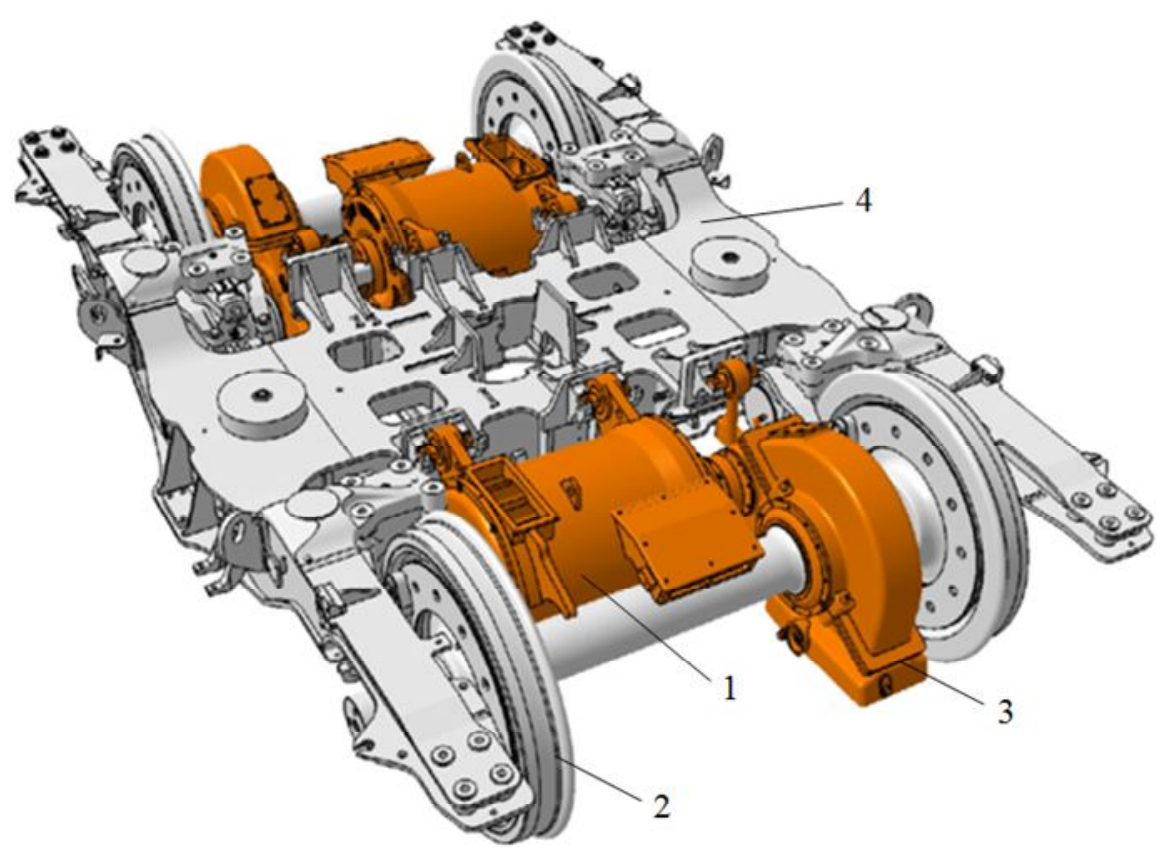

Рис. 2. Моторная тележка дизель-поезда ДЭЛ-02:

1 - тяговый асинхронный двигатель, 2 - колёсная пара, 3 - редуктор, 4 - рама

\section{Основная часть}

Задаче синтеза математических моделей электромеханической системы электропривода посвящено множество работ [3-6]. Однако, практический интерес представляет исследование электромеханической системы ТАП на математической модели, которая учитывает реализацию момента вращения ТАД при взаимодействии КП с рельсами и описывается системой дифференциальных уравнений высокого порядка. При этом, удовлетворительные результаты можно получить и на более простой модели $[7,8]$, которая описывается системой обыкновенных дифференциальных уравнений:

$$
\begin{gathered}
\frac{\mathrm{d} \omega_{1}}{\mathrm{dt}}=\frac{1}{\mathrm{~J}_{1}}\left(\mathrm{iM}_{\text {эл }}-\mathrm{k}\left(\mathrm{U}_{\delta}, \mathrm{V}_{\mathrm{t}}\right) \Psi_{0} \Pi_{\mathrm{k}} \mathrm{R}_{\mathrm{k}}\right)+\delta_{1}(\mathrm{t}), \\
\frac{\mathrm{d} \omega_{2}}{\mathrm{dt}}=\frac{1}{\mathrm{~J}_{2 д}}\left(\begin{array}{c}
\mathrm{c}\left(\omega_{1}-\omega_{2}\right)+\mathrm{b}\left(\phi_{1}-\phi_{2}\right)- \\
-\mathrm{k}\left(\mathrm{U}_{\delta}, \mathrm{V}_{\mathrm{t}}\right) \Psi_{0} \Pi_{\mathrm{k}} \mathrm{R}_{\mathrm{k}}
\end{array}\right)+\delta_{2}(\mathrm{t}),(1) \\
\frac{\mathrm{d} \phi_{1}}{\mathrm{dt}}=\omega_{1}, \frac{\mathrm{d} \phi_{2}}{\mathrm{dt}}=\omega_{2},
\end{gathered}
$$

где $\omega_{1}, \omega_{2}-$ угловые скорости шестерни и колесной пары соответственно; $\delta_{1}, \delta_{2}-$ случайные возмущения; $\mathrm{J}_{1 д ~}$ - моменты инерции ротора ТАД, шестерни, редуктора, полого вала, которые приведены к оси КП; $\mathrm{J}_{2 д}$ - момент инерции КП; $\mathrm{i}$ - передаточное число редуктора; $\mathrm{M}_{\text {эл }}$ - электромагнитный момент ТАД; $\mathrm{k}\left(\mathrm{U}_{\delta}, \mathrm{V}_{\mathrm{t}}\right)$ - нелинейный коэффициент, зависящий от скорости дизель-поезда $\mathrm{V}_{\mathrm{t}}$ и скорости боксования $\mathrm{U}_{\delta} ; \Psi_{0}$ - потенциальное значение коэффициента сцепления КП с рельсом; $\Pi_{\mathrm{k}}-$ текущее значение сцепного веса КП; $\mathrm{R}_{\mathrm{k}}$ - радиус колес КП; $\phi_{1}, \phi_{2}$ - углы поворота шестерни и КП; с - жесткость упругих связей в системе привода; $\mathrm{b}-$ коэффициент рассеивания энергии колебаний упругих связей; $\mathrm{M}_{\text {сц }}=\mathrm{k}\left(\mathrm{U}_{\delta}, \mathrm{V}_{\mathrm{t}}\right) \Psi_{0} \Pi_{\mathrm{k}} \mathrm{R}_{\mathrm{k}}-$ момент сцепления КП с рельсом.

Анализ результатов, полученных на математической модели (1), подтвердил наличие фрикционных колебаний в электромеханической системе ТАП дизель-поезда. Поскольку конструктивное исполнение механической части привода не подлежит изменению, возникла необходимость в разработке программных средств для сглаживания колебаний частоты ДЧВ. Был проведён анализ различных способов сглаживания. Так, сглаживание колебаний частоты с помощью её усреднения по нескольким импульсам или на определённом временном интервале (2) неприемлемо из-за снижения точности и быстродействия, что равноценно уменьшению числа зубьев шестерни ДЧВ.

$$
\mathrm{x}_{\mathrm{i}}=\frac{1}{\mathrm{~m}} \sum_{\mathrm{j}=-\frac{\mathrm{m}}{2}}^{\frac{\mathrm{m}}{2}-1} \mathrm{x}_{\mathrm{i}+\mathrm{j}}
$$

где $\mathrm{x}_{\mathrm{i}}$ - показание датчика частоты в момент времени $\mathrm{i} ; \mathrm{m}$ - величина временного интервала.

Приемлемым оказалось сглаживание частоты ДЧВ путём вычисления простого скользящего среднего, которое численно равно среднему арифметическому значений за установленный период:

$$
\mathrm{X}_{\mathrm{t}}=\frac{1}{\mathrm{n}} \sum_{\mathrm{i}=0}^{\mathrm{n}-1} \mathrm{p}_{\mathrm{t}-\mathrm{i}}=\frac{\mathrm{p}_{\mathrm{t}}+\mathrm{p}_{\mathrm{t}-1}+\ldots+\mathrm{p}_{\mathrm{t}-\mathrm{n}+1}}{\mathrm{n}},
$$


где $\mathrm{X}_{\mathrm{t}}$ - значение простого скользящего среднего в точке $\mathrm{t} ; \mathrm{n}$ - количество значений исходной функции для расчёта скользящего среднего (сглаживающий интервал); $\mathrm{p}_{\mathrm{t}-\mathrm{i}}-$ значение исходной функции в точке $\mathrm{t}-\mathrm{i}$

Для случая измерения частоты с ДЧВ в качестве исходной функции используется период времени между соседними импульсами $\mathrm{t}_{\mathrm{i}}$, тогда скользящее среднее значение периода может быть получено следующим образом:

$$
\mathrm{X}_{\mathrm{t}}=\mathrm{X}_{\mathrm{t}-1}-\frac{\mathrm{p}_{\mathrm{t}-\mathrm{n}}}{\mathrm{n}}+\frac{\mathrm{p}_{\mathrm{t}}}{\mathrm{n}},
$$

где $\mathrm{X}_{\mathrm{t}}$ - значение простого скользящего среднего в точке $\mathrm{t} ; \mathrm{X}_{\mathrm{t}-1}$ - предыдущее значение простого скользящего среднего; $\mathrm{p}_{\mathrm{t}-\mathrm{n}}$ - значение периода в точке $\mathrm{t}-\mathrm{n} ; \mathrm{p}_{\mathrm{t}}$ - значение периода в точке $\mathrm{t}$.
Тогда частота $\mathrm{f}_{\mathrm{i}}$ сглаживаемых импульсов, которая соответствует частоте вращения ротора ТАД, будет равна:

$$
\mathrm{f}_{\mathrm{t}}=\frac{\mathrm{K}}{\mathrm{X}_{\mathrm{t}}} .
$$

На рис. 3 приведено изменение сигналов с датчиков частоты вращения двух тяговых асинхронных двигателямией, полученных в процессе проведения наладки тяговых приводов $\left(\mathrm{n}_{1}, \mathrm{n}_{2}-\right.$ количество импульсов в секунду с ДЧВ1 и ДЧВ2 соответственно).

Характер изменения данных сигналов свидетельствует о наличии колебаний тяговых приводах и соответствует колебаниям частоты на выходах датчиков частоты вращения, что подтверждает результаты исследования процессов в системе дизельпоезда на математической модели.

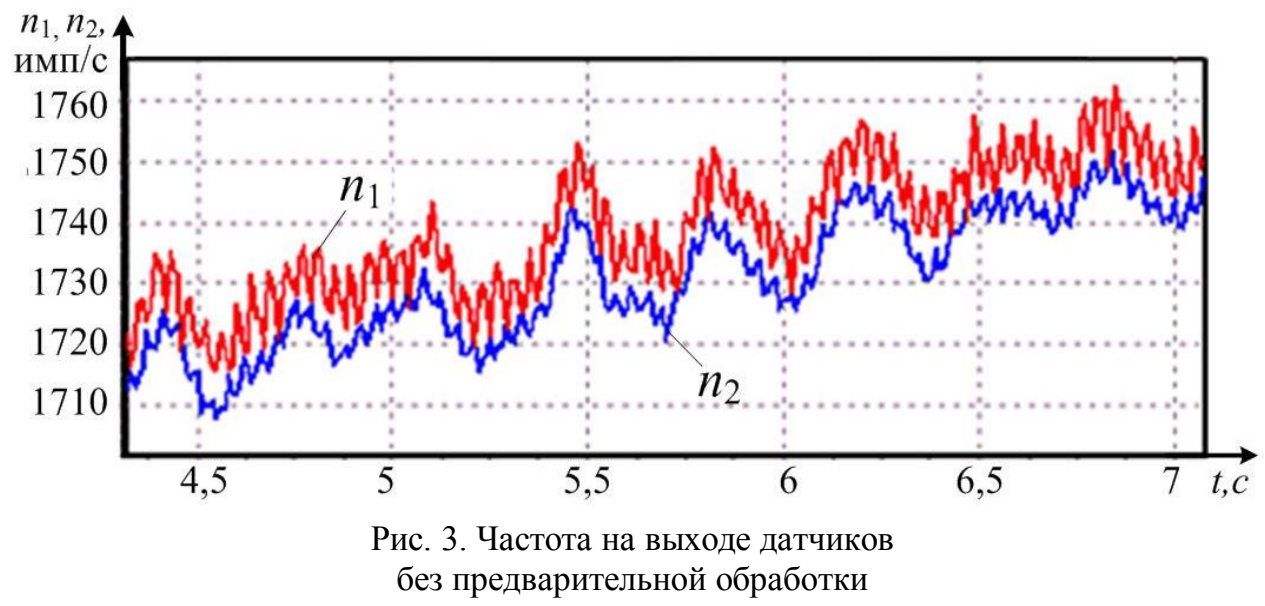

При использовании в САР тяговых приводах сглаживания частоты методом простого скользящего среднего информация о частоте вращения ротора ТАД получается с приемлемой точностью и качеством.

Достаточно просто может быть получен параметр ускорения локомотива путём дифференцирования частоты:

$$
\mathrm{a}=\frac{\mathrm{df}_{\mathrm{t}}}{\mathrm{dt}}
$$

Ускорение относится к числу основных параметров дизель-поезда, которые проверяются при его сдаче в эксплуатацию.

Использование метода простого скользящего среднего на дизель-поезде ДЭЛ-02 позволило реализовать все требования предъявляемые к ТП с компьютеризованной системой автоматического регулирования в части статических и динамических характеристик.

В частности, на рис. 4 представлено изменение сигналов ускорения дизель-поезда, а также частоты вращения ротора ТАД1, вычисленные бортовой САР ТП с использованием метода простого скользящего среднего.

Как видно из рис. 4, сигналы имеют гладкую форму и могут быть использованы для реализации в других подсистемах САР.

\section{Выводы}

Исследования на математической модели системы привода отечественного дизель-поезда ДЭЛ-02 подтвердили наличие фрикционных колебаний, которые приводят к колебаниям частоты на выходе датчика.

Проведя анализ различных способов сглаживания сигналов, получаемых с датчиков частоты вращения двигателей в тяговом асинхронном приводе, оказалось, что наиболее приемлемым является сглаживание сигналов путём вычисления простого скользящего среднего.

Описанный метод обработки сигналов был использован в САР электропередачи украинского дизель-поезда ДЭЛ-02. 


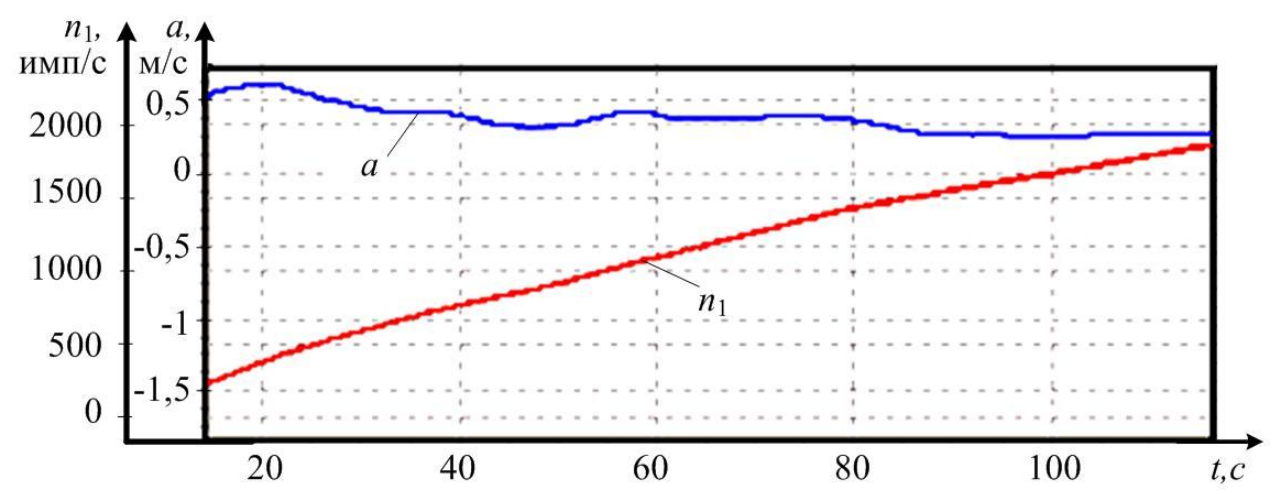

Рис. 4. Изменение сигналов ускорения дизель-поезда (а) и частоты вращения ротора ТАД1 $\left(\mathrm{n}_{1}\right)$ с предварительной обработкой

\section{Список литературы}

1. Зубенко Д.Ю. Исследование с помощью математических моделей тяговых асинхронных электроприводов скоростных электропоездов / Д.Ю. Зубенко // Коммунальное хозяйство городов. Научно-технический сборник. - Х.: 2007. - №7 - C. $337-344$.

2. Хворост М.В. Тяговий асинхронний електропривод для міського електротранспорту / М.В. Хворост, М.I. Шпіка, A.I. Бесараб // Энергосбережение. Энергетика. Энергоаудит. - 2012. - №3. - С. 7 - 10.

3. Орловский И.А. Учёт упругих связей и распределённой нагрузки при векторном управлении асинхронным тяговым приводом дизель-поезда / И.А. Орловский, А.Н. Кулешов // Вісник Дніпропетровського начіонального університету залізничного транспорту імені академіка $B$. Лазаряна. - 2007. - Bun. 19. - С. 209-213.

4. Глазырин А.С. Математическое моделирование электромеханических систем, аналитические методы: учебное пособие / А.С. Глазырин. - Томск: изд. Томского политехнического университета. - 2009. - 216 c.

5. Федяева Г.А. Моделирование перспективного маневрового тепловоза с векторным управлением асинхронным тяговым приводом / Г.А. Федяева // Вест. Брянского техн. ун-та. - 2007. - №3 (15). - C. $39-45$.
6. Федяева Г.А. Математическое моделирование электромеханических прочессов в асинхронном тяговом приводе тепловоза ТЭМ21 / Г.А. Федяева, В.Н. Федяев // Вестник ВНИИЖТ. - 2005. - №6 - С. 39 - 45.

7. Заполовский Н.И. Моделирование тягового электропривода дизель-поезда с учетом сиепления колесных пар с рельсами / Н.И. Заполовский, С.И. Червонный, Р.М. Грыб // Вестник НТУ «ХПИ». Сборник научных трудов. Серия: Информатика и моделирование. - Харьков: НТУ «ХПИ». -2006 . - №23. - C. $61-66$.

8. Носков В.В. Моделирование электромеханической системы моторного вагона с учетом диссипативных сил / В.И. Носков, А.Ю. Заковоротный, Н.В. Мезенцев, Г.В. Гейко // Автоматизированные технологии и производства. - Изд. МГТУ им. Г.И.Носова. - 2016. - №3 (13) C. $31-36$.

Надійшла до редколегії 30.01.2018

Рецензент: д-р техн. наук, проф. Б.Т. Кононов, Харківський національний університет Повітряних Сил імені Івана Кожедуба, Харків.

\title{
ОБРОБКА СИГНАЛІВ \\ У БОРТОВІЙ СИСТЕМІ УПРАВЛІННЯ ДИЗЕЛЬ-ПОЇЗДУ ДЕЛ-02
}

\author{
В.І. Носков, Н.В. Мезенцев, Г.В. Гейко, В.І. Панченко
}

Показано, щуо наявність пружних зв'язків в системі передачі моменту, а також постійно змінюючихся умов зчеплення колеса з рейкою призводять до коливань на виході датчика частоти обертання ротора. Проведено аналіз різних способів згладжування цих сигналів. Прийнятним виявилося згладжування сигналів шляхом обчислення простого ковзного середнього. Пропонується обробка сигналів, які надходять з датчиків частоти обертання двигунів в тяговому асинхронному приводi.

Ключові слова: датчик частоти обертання, тяговий асинхронний привід, система передачі моменту, обробка сигналів.

\section{PROCESSING OF SIGNALS \\ IN THE AIRBOARD CONTROL SYSTEM OF DIESEL-TRAIN DEL-02}

V.I. Noskov, N.V. Mezentsev, G.V. Geiko, V.I. Panchenko

It is shown that the presence of elastic bonds in the torque transmission system, as well as the constantly changing conditions for the adhesion of the wheel to the rail, lead to oscillations at the output of the rotor speed sensor. The analysis of various ways of smoothing of these signals is carried out. The smoothing of signals by computation of the simple moving average was acceptable. It is proposed to process signals received from the engine speed sensors in the traction asynchronous drive.

Keywords: speed sensor, traction asynchronous drive, torque transmission system, signal processing. 